\title{
Das Jahr 2013 im Palmengarten
}

\author{
Matthias Jenny \& Manfred Wessel
}

Mit frischer Kraft und wieder einem vielseitigen und abwechslungsreichen Programm startet der Palmengarten im Januar in die neue Saison 2013. Damit auch ortsfremde Besucherinnen und Besucher sich schnell über den Palmengarten informieren können, gibt es einen handlichen Palmengartenführer, der 2013 nun in der dritten, neu bearbeiteten Auflage erhältlich ist. Der Palmengarten hat immer Saison und ist während des ganzen Jahres ein Publikumsmagnet. Der Festsaal des gerade erst im Vormonat offiziell eröffneten Gesellschaftshauses wird gut angenommen; regelmäßig finden hier nun Abendveranstaltungen, Tagungen und Empfänge statt. Auf hohem Niveau angelegt ist das Restaurant Lafleur im Gesellschaftshaus. Kritiker merken allerdings an, dass das Gesellschaftshaus, wie es nun betrieben wird, leider kein Ort mehr für Frankfurter Bürger(vereine) ist. Auch im Ortsbeirat wird über das teure Gesellschaftshaus debattiert. Im Sommer aber beispielsweise wird Michael QuAst viele Zuschauer im Festsaal zu seinem "Rendevous im Palmengarten“ einladen. Im Zuge der Restaurierung des Gesellschaftshauses wurde auch die Eingangssituation im Bereich Palmengartenstraße verändert, sodass das Blumenparterre frei zugänglich ist. Das neue Kassenhaus wird in Betrieb genommen.

Es ist nun schon der dritte kräftige Winter in Folge. Zunächst ist es Anfang Januar zwar fast frühlingshaft warm, sodass klassische Winterblüher wie Kamelien und Zaubernüsse zu Beginn des Jahres einen üppigen Blütenflor zeigen. Doch Meteorologen haben Frost, Eis und Schnee angekündigt, die dann auch nicht mehr lange auf sich warten lassen. Vorwitzige Blüten erfrieren, aber der Palmengarten wirkt unter der hohen Schneedecke märchenhaft schön,

Abb. 1: Tief verschneiter Palmengarten. Im Tropicarum ist es aber auch im Winter tropisch warm.

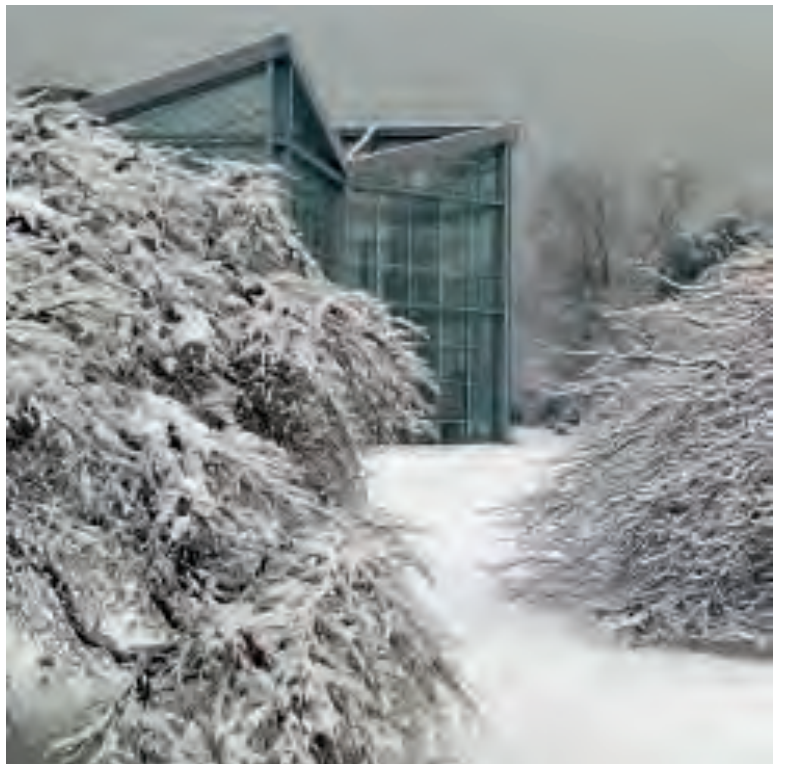

auch wenn winterlicher Sonnenschein wochenlang fehlt. Eine ganz besondere Atmosphäre verleiht die weiße Pracht der Veranstaltung „Winterlicht“, die noch bis Ende Januar verlängert wird. Durch die Lichtreflexionen an den schneebeladenen Zweigen der Gehölze wird die märchenhafte Stimmung im Garten noch verstärkt. Zwischenzeitliches Tauwetter und ein Eisregen, der im Freien alles mit einer glitzernden Eisschicht überzieht, bietet den Winterlicht-Fotografen einzigartige Motive. Zum Aufwärmen gibt es in der Galerie heißen marokkanischen Minztee, Glühwein und kleine Imbisse.

Als erste Blumenausstellung des Jahres findet die Kamelienausstellung stets große Beachtung. Ergänzt wird die Blumenschau durch Informationstafeln zur Geschichte des Gesellschaftshauses. Wegen der Kälte und der fehlenden Sonnenstrahlen wollen sich die Knospen der Kamelien leider kaum öffnen, sodass gegen Ende der Ausstellung viele Exemplare immer noch nur grün sind und ihre farbintensiven Blüten in den Knospen verstecken.

Kaum ist die Kamelienschau vorbei, lockt der Palmengarten den Frühling herbei. Wäh- 


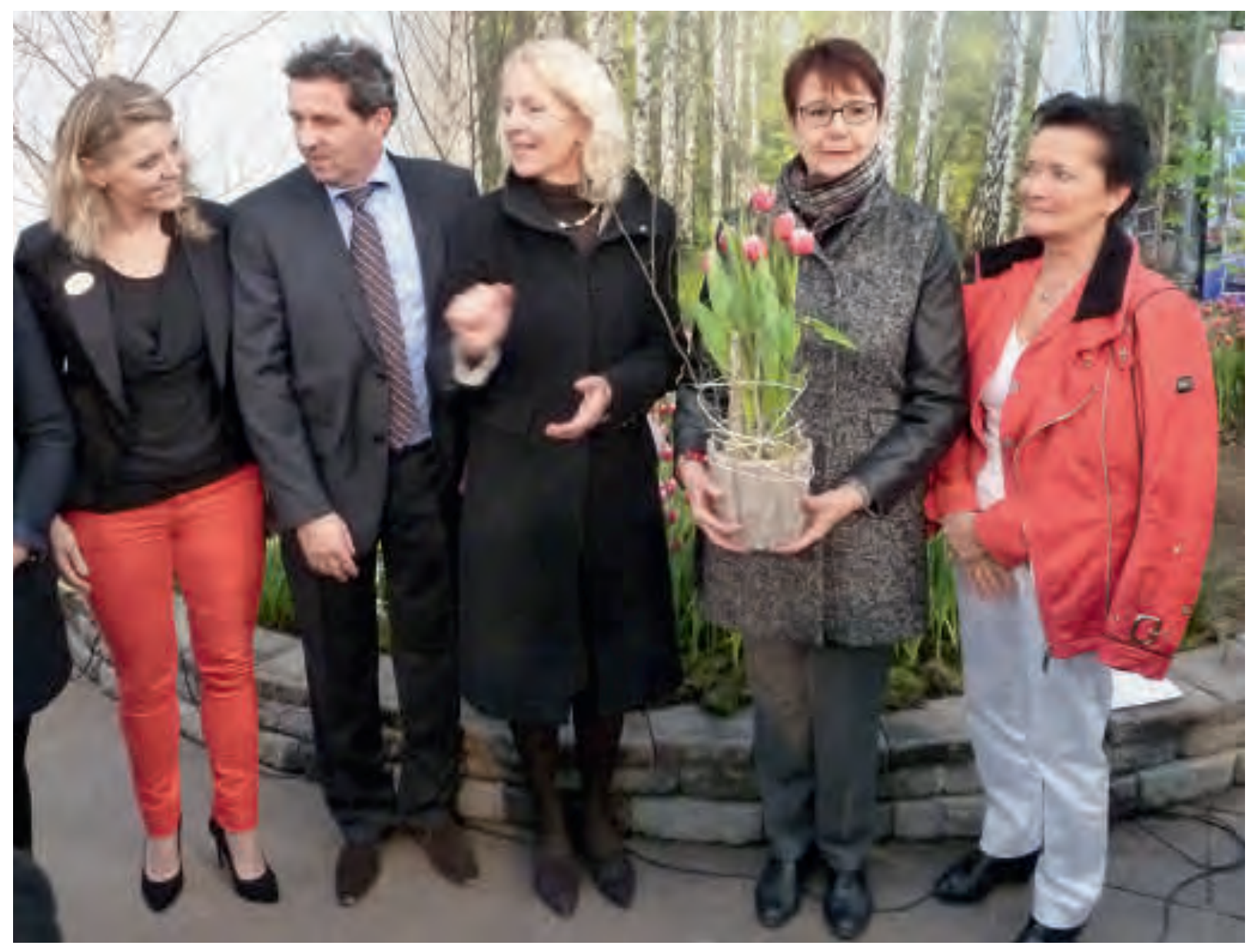

rend im Februar Krokusse und andere Geophyten unter einer Schneedecke schlummern, erblühen in der Galerie Zehntausende Tulpen und Narzissen, die extra für die Frühlingsblumenausstellung in unserer Gärtnerei vorgetrieben wurden. Diesmal erblüht die neue Sorte 'Frankfurter Frühling', eine Tulpensorte in den Frankfurter Wappenfarben Weiß und Rot. Dezernentin Rosemarie Heilig und Stadtverordnetenvorsteherin Bernadette Weyland taufen die Tulpe feierlich auf ihren Namen. Der niederländische Keukenhof und der Königliche Hoflieferant UittenbogaArd \& Zonen haben die Tulpe der Stadt gewidmet. Im kommenden Jahr soll sie auch an anderen öffentlichen Plätzen der Stadt zu sehen sein. Immer wieder ist Neues geplant. Es ist beschlossene Sache, dass ab Mai an der Lärmschutzwand zur Miquelallee hin probeweise vier verschiedene Flächen im Stil von Patric Blanc vertikal begrünt werden. Die kommenden Monate und vor allem der folgende Winter sollen zeigen, ob sich das
Projekt eines langen vertikalen Gartens realisieren lässt. Um einen so vielgestaltigen Garten wie den Palmengarten unterhalten zu können, bedarf es viel Geld. Ende Februar beschließt das Dezernat, die Eintrittspreise für den Palmengarten, wie auch für andere städtische Einrichtungen wie Museen, Schwimmbäder oder Städtische Bühnen, zu erhöhen. Ab Sommer müssen Erwachsene 7 statt 5 Euro Eintritt entrichten.

Ende Februar ist es noch einmal richtig winterlich mit viel Schnee. Nichts destotrotz öffnet der Botanische Garten am 24.2. seine Pforten für die kommende Saison.

Im März schaut der Palmengarten geschäftig seiner traditionellen Gartenmesse entgegen, viel ist kurz vorher noch zu organisieren und vorzubereiten. Es wird im März plötzlich wieder kalt und pünktlich zur Gartenmesse versinkt der Palmengarten im Schnee. Die Boote, die bereits auf dem großen Weiher einsatzbereit liegen, sind völlig eingeschneit, ein sehr ungewöhn- 
liches Bild. Aussteller und Mitarbeiterinnen und Mitarbeiter des Palmengartens müssen kräftig gemeinsam anpacken und den Schnee von Wegen und Rasenflächen schippen, damit die Ausstellerstände überhaupt aufgebaut werden können. Das Subantarktishaus wird geschlossen, weil Eiszapfen vom Glasdach abzubrechen drohen. Viele Verkaufsobjekte versinken im Schnee, und einige Pflanzen sind auch erfroren. Wegen der Kälte und des Schnees kommen deutlich weniger Besucher als sonst zur Gartenmesse. Der Festsaal ist in die Ausstellung integriert mit Informationen über Energienutzung und -Einsparung. Er wird wie die Messe abends planmäßig um 18 Uhr geschlossen. Als Scherz wurde von einer Gruppe aus der linken autonomen Szene das Gerücht verbreitet, dass es einen Tag der offenen Tür im Gesellschaftshaus gäbe und der Festsaal bis in die Abendstunden geöffnet sei. An dem entsprechenden Tag kommt es trotz der auch in der Presse veröffentlichten Falschmeldung nicht zu unangenehmen Zwischenfällen. Nur die Preispolitik des Gesellschaftshauses wird kritisiert.

Die Azaleenausstellung zeigt sich mit roter, weißer und rosa Blütenpracht, diesmal ergänzt mit Glasskulpturen von William Greenwood. Ostern mit dem bei Kindern so beliebten Osterhasengärtchen folgt. Ohne die Technik des Gartens gäbe es keine tropische Wärme für unsere Pflanzen. Auch hier gibt es Neuerungen, denn das Tropicarium wird von nun an mit Biogas beheizt. Frankfurts erstes Biokraftwerk verwertet nur organische Reststoffe wie Klärschlamm und Abfälle. Der Palmengarten hat neue Freunde und Förderer gewonnen. Die staatliche Förderbank KfW hat eine eigene Stiftung gegründet, mit der sie künftig neben

Abb. 2 (Seite 4): Eröffnung der Frühlingsblumenausstellung und Taufe der Tulpe 'Frankfurter Frühling'.

Abb. 3 (oben): Azaleenausstellung mit Glaskunst.

Abb. 4 (Mitte): Gemeinsam wird der Schnee geräumt, damit die Stände nach dem erneuten Wintereinbruch für die Gartenmesse überhaupt aufgebaut werden können.

Abb. 5 (unten): Eröffnung der SenckenberG-

Ausstellung im Botanischen Garten.
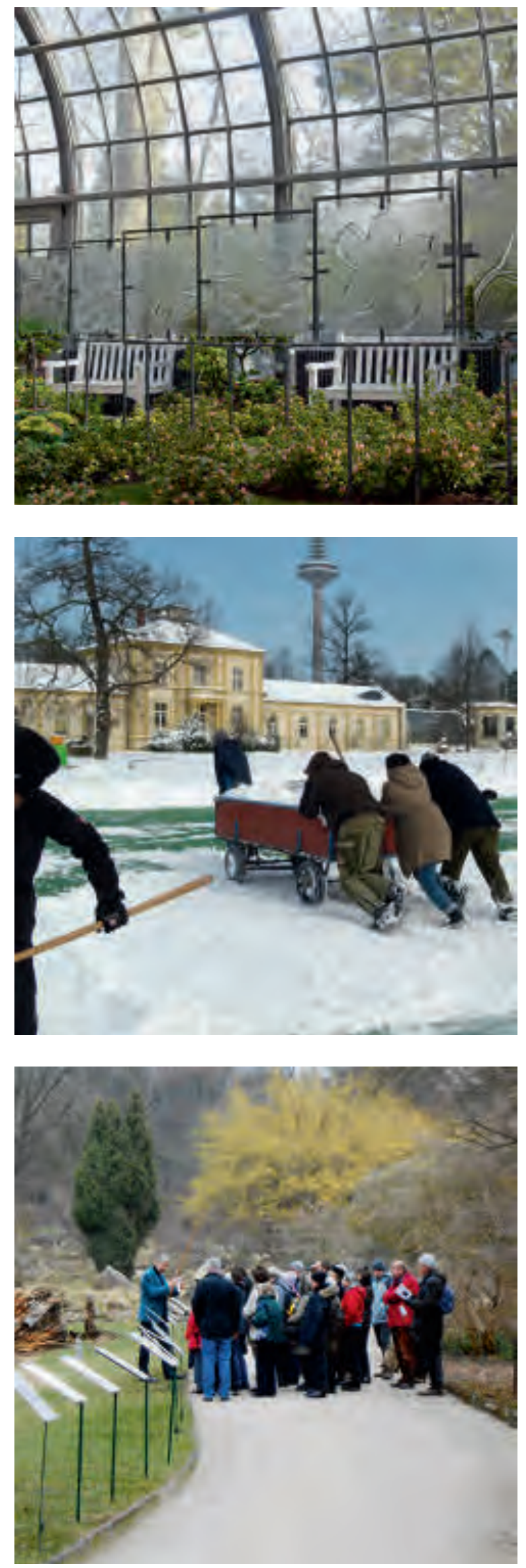

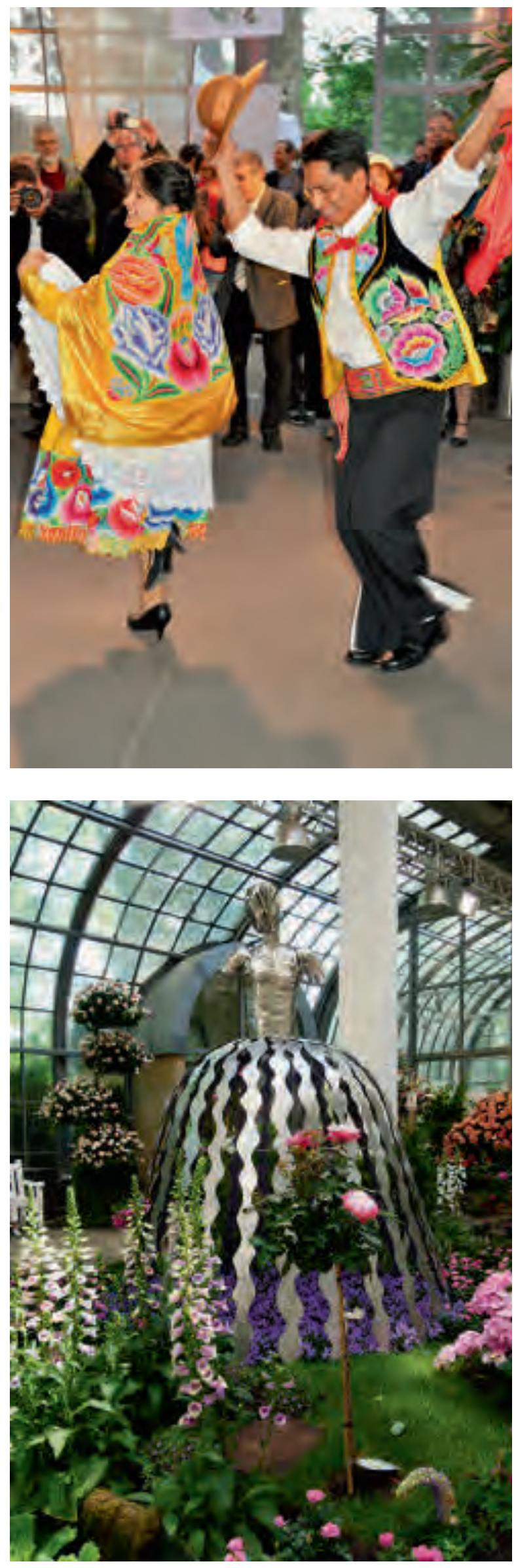

Zoo und Senckenberg-Museum auch den Palmengarten unterstützen möchte.

Ein stets stark frequentiertes Ereignis ist im April die Orchideen-Verkaufsausstellung. Mit diversen Aktivitäten geht es auch im Botanischen Garten in die Saison. Aus Anlass des 250. Geburtstages der Senckenbergischen Stiftung zeigt der Garten als eine der Gründungsinstitutionen eine Jubiläumsausstellung von den Anfängen des Hortus medicus am Eschenheimer Tor bis zum heutigen Garten. Als Ergänzung sind auch für den weiteren Verlauf des Jahres Veranstaltungen zum Thema SenckenBerg geplant. Petrus meint es mit der historischen Inszenierung im Mai leider nicht so gut, denn es regnet in Strömen. Dennoch sind Johann Christian Senckenberg und seine erste Frau Rebecca für die Veranstaltung wiederauferstanden und plaudern über Frankfurts Historie.

Im Mai ist Pflanzzeit. Dementsprechend ist der Andrang auf der traditionell Mitte Mai stattfindenden Pflanzenbörse im Botanischen Garten auch immer sehr groß. Es kommen viele Interessierte, um sich über diverse grüne Themen zu informieren. Auf der vom Freundeskreis des Botanischen Gartens organisierten Börse gibt es immer viele besondere Gewächse für den eigenen Garten zu erstehen. Grün geht es auch beim Frankfurter Grüne-Soße-Festival (11.- 18. Mai) zu, an dem sich der Palmengarten beteiligt. Am 16. Mai wird die diesjährige große Informationsausstellung „Aus der Neuen Welt“ eröffnet. Zur abendlichen Eröffnung führt eine peruanische Tanzgruppe, gekleidet in farbenfrohen Trachten, schwungvolle Volkstänze auf. In der Ausstellung geht es um essbare Nutzpflanzen, die bei uns verwendet oder sogar angebaut werden, ihre Heimat aber in Amerika haben. Für ausgewählte Regionen aus Nordund Südamerika werden jeweils repräsentative

Abb. 6 (oben): Eröffnung der Ausstellung „Aus der Neuen Welt" mit peruanischem Volkstanz.

Abb. 7 (unten): Rosenausstellung 2013.

Abb. 8 (Seite 7): Teelicht-Rose während des Rosenund Lichterfestes. 
Pflanzen mit ihrer Einführungsgeschichte vorgestellt, darunter auch Kartoffel, Mais und Tomate. Ergänzend dazu ist ein „Indianerbeet“ im Botanischen Garten angelegt, auf dem sich Sonnenblumen, Erdnusspflanzen, Bohnen, Mais sowie verschiedene Kartoffel- und Tomatensorten gut entwickeln können. Ein umfangreiches Rahmenprogramm, so z. B. auch ein Tomatenwochenende, bereichern die von vielen besuchte Ausstellung. Wegen des nasskalten Frühjahres ist die Vegetationsentwicklung auch jetzt noch verzögert.

So ist der Rosengarten Anfang Juni noch ganz grün, während normalerweise um diese Zeit die Rosen meist prächtig aufgeblüht sind. In diesem Jahr blüht so gut wie keine Freilandrose zur Eröffnung der Rosenschau. Dafür ist aber die Galerie besonders üppig mit 5000 Schnittund 700 Topfrosen gestaltet. Harmonisch fügt sich zwischen den Rosen eine überlebensgroße Rosenprinzessin aus Stahl ein, geschaffen von der Frankfurter Künstlerin E. R. Nele. Von der Pracht der Ausstellung können sich auch die geladenen Gäste des Dezernats-Empfangs, der in der Rosenausstellung stattfindet, überzeugen. Highlight ist am Samstagabend das Lichterfest mit dem großen Feuerwerk.

In den letzten Jahren gab es im Palmengarten häufig Wasserrohrbrüche. Es steht nun fest, dass von Seiten der Stadt Mittel für die Sanierung der Leitungen bewilligt worden sind. Die marode Ringleitung kann nun für über 5 Mio. Euro saniert werden. Das Verlegen der neuen Rohre wird in den kommenden drei Jahren jeweils in den besucherärmeren Wintermonaten erfolgen. Die neue Eingangskasse weist leider erhebliche Baumängel inklusive Schimmelbefall auf und muss geschlossen werden. Ersatzweise wird vorübergehend auf der gegenüberliegenden Seite des Blumenparterres ein Kassencontainer aufgestellt. Neuen Ärger gibt es wenig später, denn der Vorplatz an der Palmengartenstraße muss aufgerissen werden, da das elektrische Rolltor aufgrund unsachgemäßer Ausführung nicht funktioniert und nun nachgebessert werden muss.

Ab Juli ist es dann endlich richtig Sommer geworden, die vielen heißen und sonnigen Tage

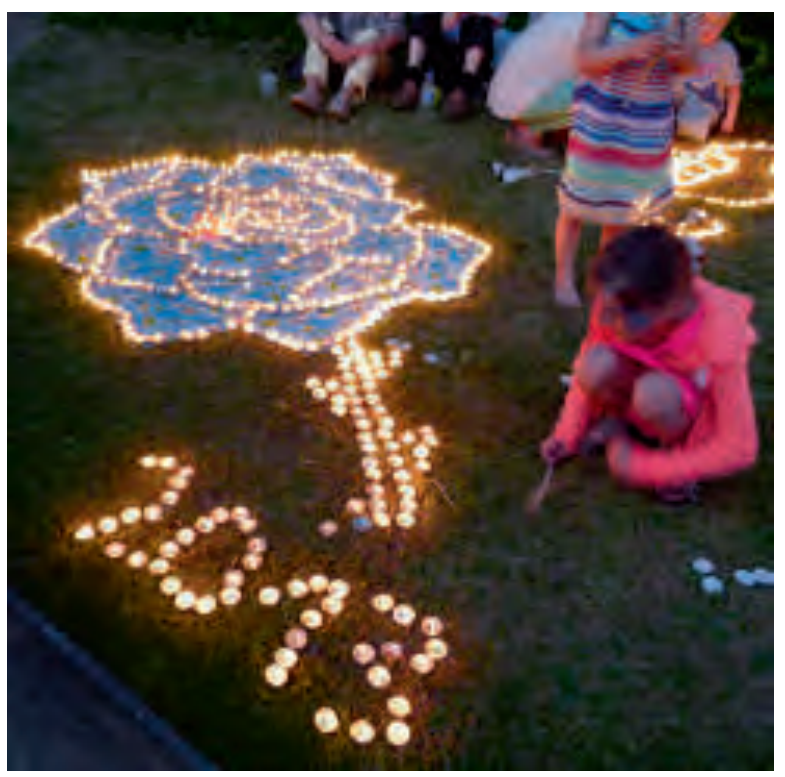

verwöhnen uns bis in den Herbst hinein und lassen den langen kalten Winter vergessen. Davon profitieren auch die vielen Freiluftveranstaltungen unseres Musikprogrammes wie Kammeroper, Jazz im Palmengarten, Weltmusik, Blues oder die Promenadenkonzerte. Viele Freunde unserer Open-Air-Konzerte genießen, oftmals mit Picknickkörben ausgerüstet, die lauen Sommerabende. Musikfreunde sind entsetzt darüber, dass die Konzertreihen des Mousonturms (Weltmusik, Jazz) aus finanziellen Gründen in der Saison 2014 gefährdet sind. Es werden viele Unterschriften zum Erhalt dieser Konzerte gesammelt, die dann auch vorerst wohl gerettet sind. In der Galerie Ost können die vielen, zum Teil überdimensional großen Bilder des Malers Wolfram SACHs bewundert werden. Neu ist in diesem Sommer auch der Biergarten („Lindengarten“) zwischen Konzertgarten und Gesellschaftshaus, in dem man sich mit Kleinigkeiten erfrischen und stärken kann.

Gärten sind grüne Oasen zum Entspannen. Sehlehrerin Christina Mundt-Beisel nutzt den Botanischen Garten, um auf einem Erlebnispfad einen Augenspaziergang anzubieten, auf dem müde Augen wieder munter werden sollen. Ihr Grundgedanke ist, dass man seine Sehkraft durch Augenübungen, Entspannung und Visualisierung verbessern und stärken kann. Es handelt sich dabei um eine ganzheitliche Methode. 

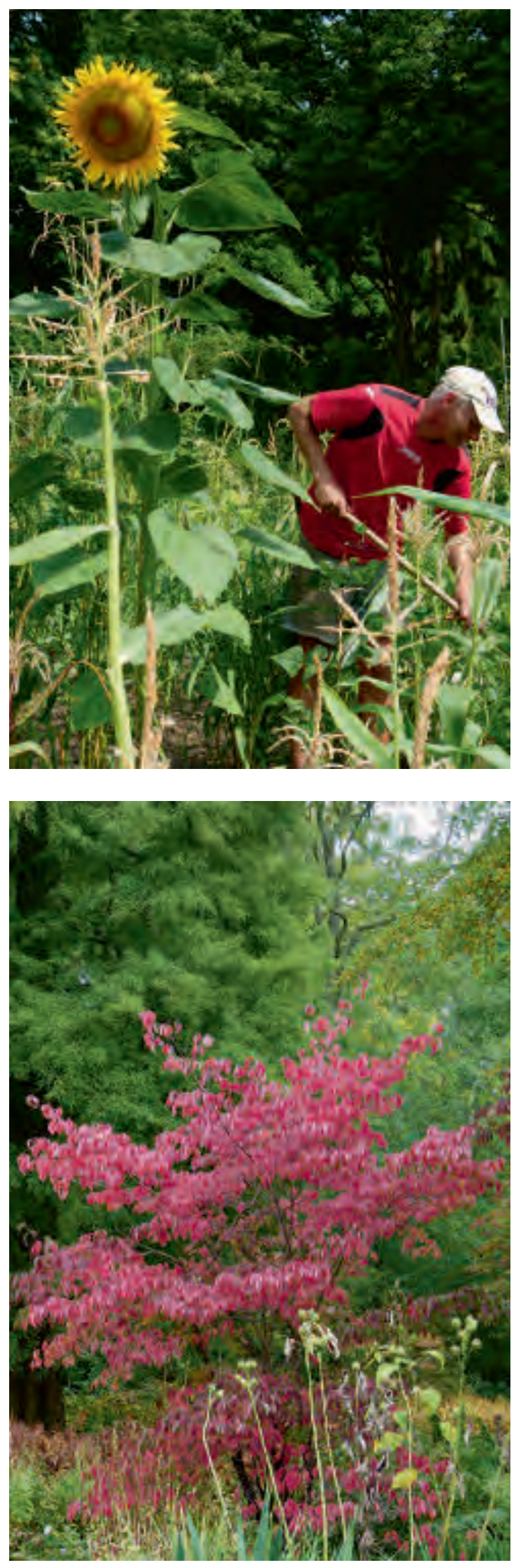

Wer im Sommer nicht in den Urlaub fährt, kann sich auch wunderbar im Palmengarten erholen. Im August sind Steppenwiese, Seerosenbecken, Sommerflor oder auch das Indianerbeet im Botanischen Garten sehr üppig entwickelt. Auch der Senckenbergische Arzneipflanzengarten ist während des ganzen Sommers ein Publikumsmagnet. Im August blüht auch wieder mal eine Titanenwurz. Der große Stinker lässt sich mit dem Aufblühen etwas Zeit. Pünktlich zum Bildzeitungsfest, und nur an diesem Abend, ist der große Blütenstand geöffnet, sodass auch prominente Gäste wie die Jacob Sisters die Möglichkeit haben, diese außergewöhnliche Pflanze zu bestaunen. Der 18. August ist ein Höhepunkt im Jahresverlauf des Botanischen Gartens. An diesem Tag findet im Casino auf dem Campus Westend der Universität mit vielen Besuchern, Ausstellungen und Vorträgen die große feierliche Festveranstaltung der Senckenbergischen Gründungseinrichtungen statt. Der Botanische Garten ist dort gebührend vertreten.

Am 7. September findet im Botanischen Garten, wie immer am ersten Samstag im September, der Tag der offenen Tür statt. Es gibt ein vielseitiges Rahmenprogram u.a. mit Führungen, Kinderprogramm und einem Blick hinter die Kulissen. Traditionell sorgen selbst gekochte Kürbissuppe oder hausgemachter $\mathrm{Ku}$ chen für das leibliche Wohl. Die Farbe Orange dominiert im herbstlichen Palmengarten, vor allem auch in der Erntedankschau. Das Herbstfest ist ein beliebtes Familienfest, bei dem u.a. jede Menge dicke Kürbisse geschnitzt oder Lampions gebastelt werden. Im ausklingenden Sommer ist nach den großen Ferien der September ein beliebter Monat für Tagungen und Straßen- bzw. Gartenfeste. In den letzten Jahren hat sich der Palmengarten mit einem Informationsstand auf dem Fürstlichen Gartenfest

Abb. 9 (oben): Indianerbeet im Botanischen Garten als Ergänzung zur „Neuen Welt“.

Abb. 10 (unten): Herbst im Botanischen Garten.

Abb. 11 (Seite 9): Eröffnung der ChrysanthemenAusstellung. 


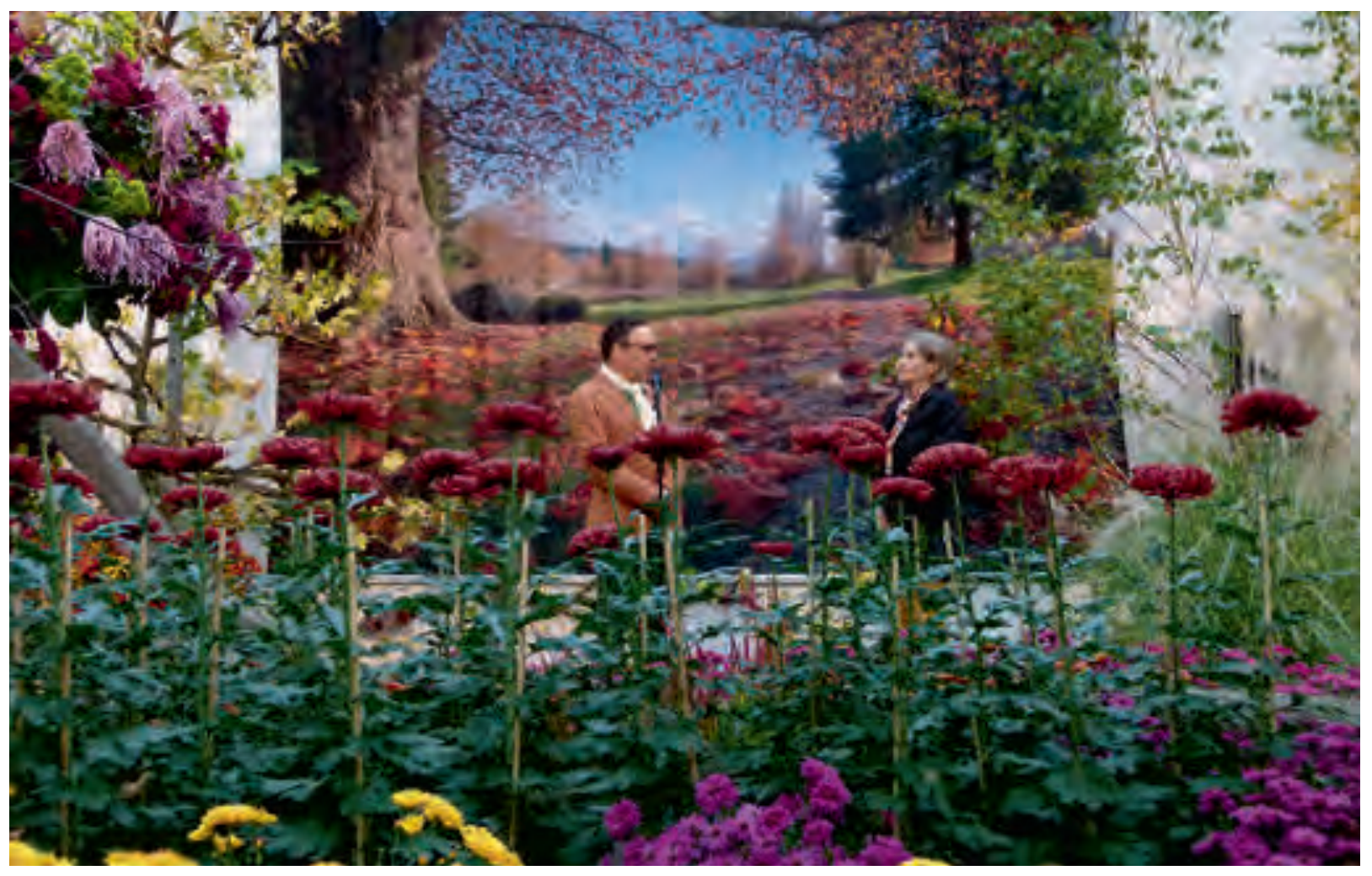

des Landgrafen von Hessen auf Schloss Wolfsgarten in Langen präsentiert. Diesmal bekommt der Palmengarten für die originelle Gestaltung des Standes zum Thema „British Gardening “ im Rahmen des Pressepreis-Wettbewerbs einen bronzenen Wolfskopf verliehen. Viel zu tun gibt es auch gegen Ende des Monats. Vom 26.-30. September findet im Palmengarten die Jahrestagung des Verbandes der Botanischen Gärten Deutschlands statt. Etwa 200 Teilnehmer aus verschiedenen Gärten sind nach Frankfurt gereist. Neben Fachvorträgen und Workshops hat der Palmengarten auch ein spannendes Rahmenprogramm organisiert, darunter auch einen Besuch des neuen Wissenschaftsgartens auf dem Campus Riedberg, der erst 2014 offiziell eröffnet werden wird. Die Gewächshauspflanzen aus dem Botanischen Garten sind gerade erst frisch in das neue Gewächshaus am Riedberg umgesiedelt. Die „Woche der Botanischen Gärten“, die bundesweit in allen deutschen Botanischen Gärten durchgeführt wird, wurde als UN-Dekade-Projekt ausgezeichnet.

Der Palmengarten nimmt am Programm „Ökoprofit" der Stadt Frankfurt teil. Durch
Einsparung von Energie sollen Geld gespart und die Umwelt geschont werden. Die neue Tiefgarage der KfW kann aus Sicherheitsgründen leider nicht, wie geplant, für Besucher des Palmengartens zur Verfügung gestellt werden.

Im Oktober wird es mit der Orchideen-Verkaufsausstellung noch einmal richtig bunt in der Galerie West im Palmengarten. Auch die anschließende Chrysanthemenausstellung ist fröhlich-bunt gehalten. Im Freiland dagegen nimmt die Blütenpracht naturgemäß allmählich ab. Ein Highlight im Botanischen Garten ist die mit dem Teestrauch verwandte Franklinia, die noch bis spät in den Herbst hinein ihre großen weißen Blüten öffnet.

Für die kühleren Tage im November ist ein Spaziergang durch das Tropicarium oder das Palmenhaus empfehlenswert, denn dort blüht immer etwas. Tropische Pflanzen und Tiere begeistern viele Menschen. So hat auch die Stiftung Palmengarten und Botanischer Garten einen Preis beim renommierten Econ Award in Berlin für ihren Kalender mit tropischen Schmetterlingen gewonnen. Der außergewöhnliche Kalender, der Kunst, Ästhetik und Information miteinander verbindet, wurde in $\mathrm{Zu}$ - 

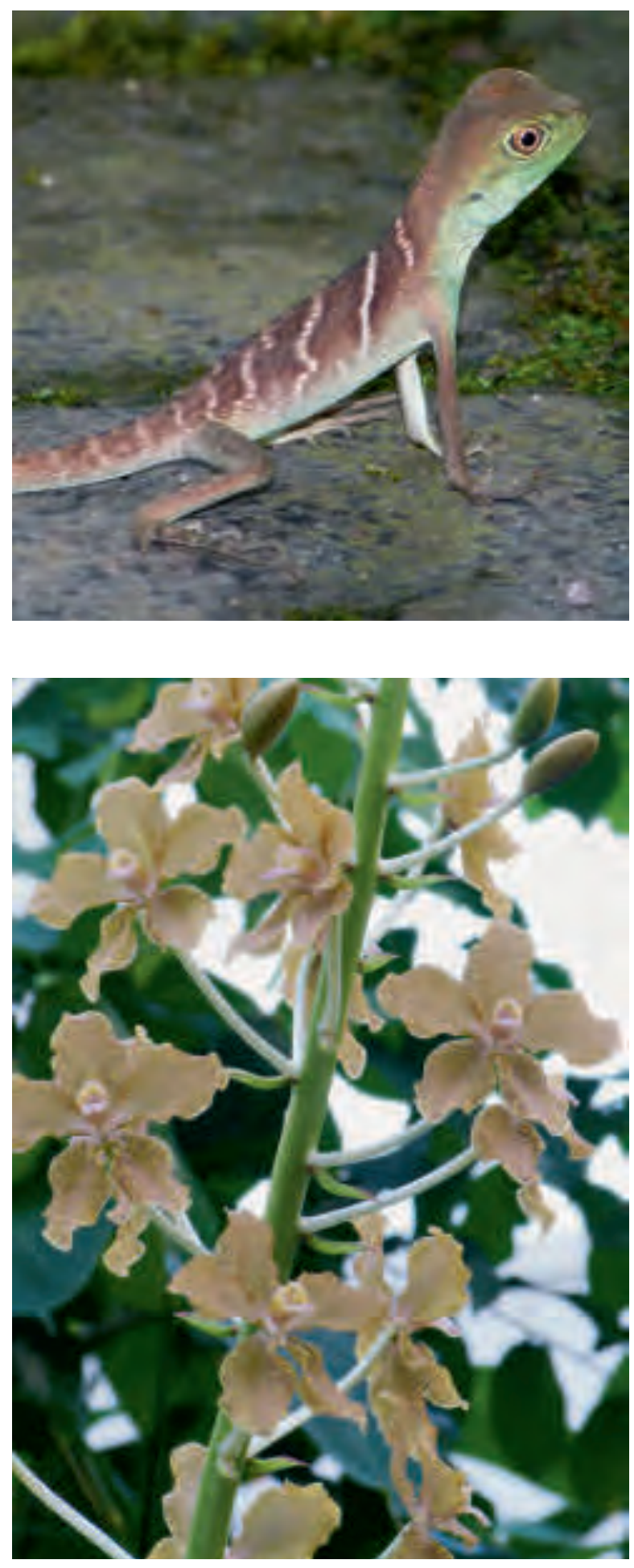

Abb. 12 (oben links): Nachwuchs bei den Wasseragamen im Tropicarium.

Abb. 13 (oben rechts): Im Rahmen der Verbandstagung wird auch das gerade fertiggestellte Gewächshaus im neuen Wissenschaftsgarten besichtigt.

Ab. 14 (unten): Nach 10-jähriger Pause blüht endlich wieder Gramatophyllum speciosum, die größte Orchidee der Welt.

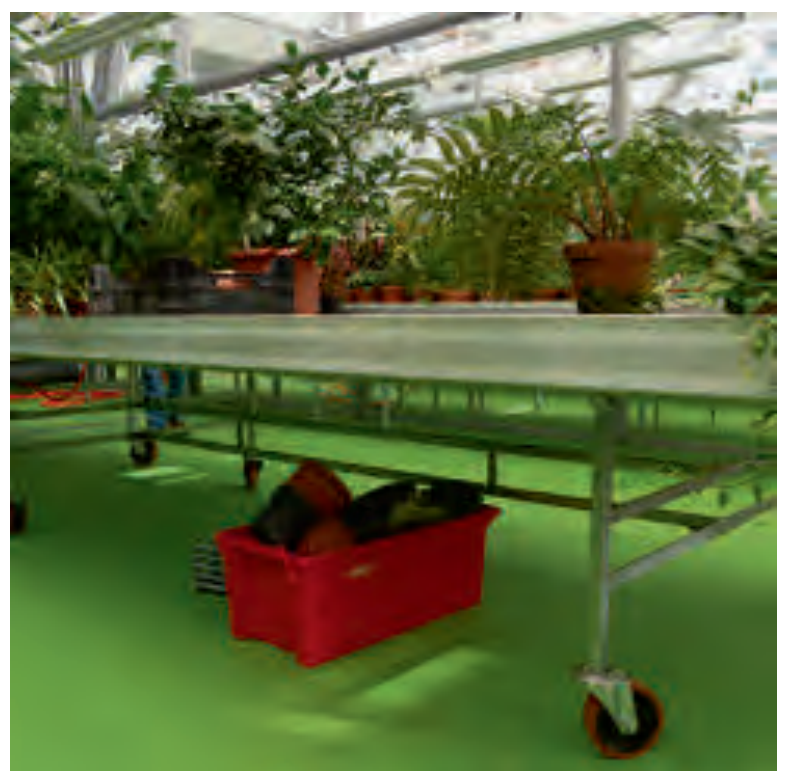

sammenarbeit mit der Agentur Hilger \& Boie Design entwickelt. Wir hoffen, dass dies wieder ein kleiner Baustein für die Realisierung des Schmetterlingshauses im Palmengarten sein wird. Sehr kreativ in eine Orangerie umgestaltet ist nun das Blütenhaus. In den nächsten Monaten werden dort fruchtende Zitruspflanzen aus der Sammlung unserer Gärtnerei gezeigt.

Vom 1. Advent bis Weihnachten verwandelt sich die Galerie West im Dezember in eine Winter- und Weihnachts-Landschaft. Neben Weihnachtssternen und festlich geschmückten Weihnachtsbäumen prägen auch von der Decke herunterhängende blühende Amaryllis das Bild. Am 14. Dezember kommen dann noch die „Winterlichter“ dazu. Der Garten ist wieder traumhaft illuminiert, von Beginn an kommen bereits deutlich mehr Besucher als im Vorjahr und alle sind begeistert; nur der Schnee bleibt leider aus. Lampen, die Schneeglöckchen nachempfunden sind, verströmen in der Winterlichter-Gastronomie in der Galerie Ost eine warme Atmosphäre. Das Jahr neigt sich dem Ende entgegen, neue Herausforderungen warten im nächsten Jahr auch wieder auf uns und werfen bereits ihre Schatten voraus. Es ist z. B. im Dezember abgemachte Sache, dass es im März im Festsaal einen Frühlingsball zugunsten der Stiftung Palmengarten und Botanischer Garten geben wird. 